\title{
Randomized Intervention Trial to Decrease Bisphenol A Urine Concentrations in Women: Pilot Study
}

\author{
Todd Hagobian, PhD, Allison Smouse, BS, Mikaela Streeter, BS, Chloe Wurst, BS, \\ Andrew Schaffner, $\mathrm{PhD}^{2}$ and Suzanne Phelan, $\mathrm{PhD}^{1}$
}

\begin{abstract}
Background: Previous studies have shown that women have higher concentrations of the endocrine disruptor bisphenol A (BPA), but an intervention to reduce BPA is lacking in women. To test the hypothesis that an intervention to reduce BPA would decrease urinary BPA concentrations over 3 weeks, 24 women (mean \pm standard deviation [SD]; $22.1 \pm 2.8 \mathrm{~kg} / \mathrm{m}^{2}$. body mass index, $20.9 \pm 1.5$ years) were randomly assigned to an intervention or control.

Materials and Methods: The intervention included weekly face-to-face meetings to reduce BPA exposures from food, cosmetics, and other packaged products. Women were provided with BPA-free cosmetics, hygiene, glass food/water containers, and daily self-monitored major sources of BPA. Fasting urine BPA and creatinine concentrations, and weight were assessed at study entry and after 3 weeks.

Results: A significant $(p=0.04)$ treatment $\times$ time interaction effect was observed on creatinine-adjusted BPA concentrations. From study entry to 3 weeks, women in the intervention significantly decreased geometric mean creatinine-adjusted urinary BPA by $-0.71 \mathrm{ng} / \mathrm{m}$, whereas women in the control significantly increased urinary BPA by $0.32 \mathrm{ng} / \mathrm{mL}(p=0.04)$. Additionally, from study entry to 3 weeks, women in the intervention significantly lost weight $-0.28 \pm 0.44 \mathrm{~kg}$, whereas women in the control significantly gained weight $+1.65 \pm 0.74 \mathrm{~kg}(p=0.03)$. Changes in creatinine-adjusted BPA concentrations and weight were not significantly related $(p=0.67)$.

Conclusion: In this pilot study, a 3-week intervention decreased urinary BPA concentrations in women. Future clinical trials are needed to confirm these results and to examine whether a similar BPA intervention positively impacts risk markers in the pathogenesis of cardiovascular disease and diabetes.
\end{abstract}

Keywords: BPA, intervention, weight, endocrine disruptors, diet

\section{Introduction}

B ISPHENOL A (BPA) is A chemical compound routinely used in the production of epoxy resins and polycarbonate plastics, appearing in the lining of food and beverage containers and several other products commonly used by consumers. ${ }^{1,2}$ Human exposure to BPA is widespread; an analysis of National Health and Nutrition Examination Survey (NHANES) data showed that $>92 \%$ of the U.S. population had detectable levels of BPA. ${ }^{3}$ Accumulating evidence suggests that high BPA concentrations are associated with negative health and weight consequences. ${ }^{4-9}$

NHANES data has also shown associations between urinary BPA concentrations and type- 2 diabetes, ${ }^{10}$ peripheral artery disease, ${ }^{9}$ metabolic syndrome, ${ }^{7}$ and obesity. ${ }^{8}$ Animal and human data indicate that BPA has estrogenic activity and may alter menstrual cycle phase length. ${ }^{11}$ However, it is important to note that not all cross-sectional studies have shown an association between BPA and negative health outcomes (e.g., obesity, type 2 diabetes). ${ }^{12,13}$ Additionally, in a reanalysis of NHANES data, LaKind et al. found no association between urinary BPA and coronary heart disease, heart attack, and diabetes, suggesting that NHANES data may be inappropriate to use in drawing conclusions about chemical exposure and negative health outcomes. ${ }^{14}$

Despite accumulating evidence associating urinary BPA concentrations with negative health and weight consequences, few studies have tested interventions to decrease BPA concentrations. Rudel et al. ${ }^{15}$ showed that a 3-day "fresh food" diet intervention reduced BPA concentrations by 
$2.6 \mathrm{ng} / \mathrm{mL}$, consistent with dietary food and beverage intake as major sources of BPA exposure. In contrast, Sathyanarayana et al. ${ }^{16}$ conducted a randomized trial on 10 families and showed that a 5-day dietary replacement intervention providing families with catered food led to a $0.8 \mu \mathrm{g} / \mathrm{L}$ increase in urinary BPA concentrations. However, the authors found post-hoc that some of the food provided had di(2-ethylhexyl) phthalate (DEHP) contamination, and then presumed BPA contamination might also have existed. To date, no study has examined the effects of a theoretically based behavioral intervention designed to lower a broad range of BPA exposures, and no known study has examined intervention effects lasting beyond 1 week.

Thus, the purpose of this pilot study was to determine the effect of a comprehensive BPA-free intervention targeting BPA exposures from food, personal hygiene products, cosmetics, and feminine hygiene products. We hypothesized that the intervention, relative to control, would decrease urinary creatinine-adjusted BPA concentrations over 3 weeks. In secondary analyses, we assessed the effects of the intervention on weight and explored relationships between changes in urinary BPA concentrations and weight.

\section{Materials and Methods}

\section{Participants}

Twenty-four, healthy, premenopausal, college-aged, normal-weight women (body mass index [BMI] $22.1 \pm 2.8 \mathrm{~kg} /$ $\mathrm{m}^{2}$ ) from California Polytechnic State University in San Luis Obispo, CA participated in this study. Eligibility included: (1) normal-weight (18.5-24.9 $\left.\mathrm{kg} / \mathrm{m}^{2} \mathrm{BMI}\right)$ women, (2) diseasefree, (3) non-smoking, and (4) exposed to multiple sources of BPA based on questionnaire (see below). Women only were included because BPA concentrations are typically higher in women than men $^{3}$; participants had to have a BMI between 18.5 and $24.9 \mathrm{~kg} / \mathrm{m}^{2}$ to ensure weight stability, ${ }^{17}$ and to examine the effects of the intervention on BPA independent of obesity. Disease and smoking status were assessed by a health history questionnaire. Women completed a modified version of a 2-day diet practices survey to identify exposure to BPA, as previously described. ${ }^{15}$ Women then completed a survey and reported use and packing of makeup products (foundation, mascara, eye shadow, etc.), hygiene products (hand sanitizer, face and body lotion, soap, shampoo, etc.), and feminine products (tampons, pads), to identify nondietary potential sources of BPA exposure. At least 13 of 24 total products had to be packaged in plastic to be included in the study. The Institutional Review Board at California Polytechnic State University approved the study, and all women gave verbal and written consent.

\section{Protocol}

After eligibility was determined, women reported to the Kinesiology Department at California Polytechnic State University in San Luis Obispo in the morning after an overnight fast ( $8-10$ hours). Weight was measured in kilogram by a standard balance beam scale and height by a stadiometer to the nearest centimeter. Women then gave a fasting urine sample for BPA and creatinine concentrations. Women were then randomly assigned, by a computer-generated program, to control group or 3-week intervention group to decrease
BPA concentrations. Randomization was concealed, and research assistants were "blinded" to treatment group assignment. Women in the control group received a weekly email newsletter that provided general information about BPA, healthy eating, and physical activity. Women in the intervention group received all aspects of the control group, plus a behavioral intervention designed to decrease urinary BPA concentrations. The intervention promoted the use of selfregulation skills (i.e., planning, self-monitoring, problem solving, goals, self-incentives) as well as positive reinforcement and counselor feedback. The intervention included weekly face-to-face meetings with the counselor. At the first meeting, the counselor discussed the negative health consequences of BPA, and how to avoid BPA exposures through daily changes in diet and personal hygiene; strong emphasis was placed on consumption of organic foods. Women brought in their plastic and BPA-containing products, and were provided with BPA-free glass Tupperware, water bottles, makeup, hygiene, and feminine products. The make-up, hygiene, and feminine products were packaged in BPA-free plastic containers, glass and/or cardboard; this information was provided by the manufacturers, and we did not directly test BPA levels in the packaging. Participants' plastic and BPAcontaining products were returned after study completion. Women were asked to self-monitor all food and beverages and associated packaging, and recorded a daily diary of foods consumed and packing of foods (plastic, glass, cardboard, other material). At the subsequent face-to-face visits, women were provided feedback on self-monitoring records and encouraged to continue self-monitoring and avoid BPAcontaining products with the goal that all foods and drinks consumed were packaged in BPA-free glass and cardboard containers or materials. After 3 weeks, women provided the final assessment of fasting urine and weight. Women were asked to refrain from physical activity immediately before each urine collection.

\section{Urinary analysis}

Fifteen milliliters of fasting mid-stream urine was collected in a BPA-free sterile specimen container and then aliquoted into three separate BPA-free polypropylene tubes, and stored at $-80^{\circ} \mathrm{C}$ until analyzed. High-Performance Liquid Chromatography/Tandem Mass Spectrometry using isotope dilution quantification assessed quantitative assessment of urinary BPA concentrations. Urinary creatinine concentration was assessed by a colorimetric assay.

\section{Sample size calculation and statistical analyses}

The sample size calculation for this pilot study was based on the study conducted by Rudel et al.$^{15}$ that showed a 3-day "fresh food" diet intervention reduced BPA concentrations by $66 \%(3.8$ vs. $1.2 \mathrm{ng} / \mathrm{mL} ; 2.5 \mathrm{SD})$. Thus, with 24 total subjects, we had $81.7 \%$ power to detect a $-2.6 \mathrm{ng} / \mathrm{mL}$ difference in urine BPA concentrations between women in the BPA intervention versus control using an $\alpha=0.05$. In this pilot study, our original intent was to enroll and randomize 30 women (15 in each group); however, recruitment was stopped prematurely at 24 participants to preserve funding for laboratory analyses. Thus, randomization resulted in slightly unequal numbers in groups. 
Table 1. Study Entry Participant Characteristics

\begin{tabular}{llll}
\hline & Control & Intervention & $\mathrm{p}$ \\
\hline Number of participants & \multicolumn{1}{c}{11} & \multicolumn{1}{c}{13} & \\
Age (years) & $21.4(1.5)$ & $20.5(1.5)$ & 0.19 \\
Weight $(\mathrm{kg})$ & $61.2(7.4)$ & $60.7(7.3)$ & 0.88 \\
Height $(\mathrm{m})$ & $1.65(0.05)$ & $1.67(0.06)$ & 0.32 \\
Body mass index $\left(\mathrm{kg} / \mathrm{m}^{2}\right)$ & $22.5(3.1)$ & $21.7(2.5)$ & 0.47 \\
\hline
\end{tabular}

Values are mean (SD).

SD, standard deviation.

A commercial software package from SAS (Cary, NC) was used for statistical analysis of data. Study entry participant characteristics were compared using a two-sample $t$-test. Raw BPA concentrations were not normally distributed, and were log transformed for statistical analyses. Similar to Barr et al. ${ }^{18}$ and Rudel et al., ${ }^{15}$ we adjusted for urinary creatinine concentrations as a fixed effect in our statistical model. A repeated measures analysis of variance (RMANOVA) was used to examine the effects of treatment group (control vs. intervention) on log-transformed urinary BPA concentrations over time, using urinary creatinine concentrations and BMI as fixed effect, and adjusting (covariate) for study entry BPA concentrations. Similar to other studies and NHANES values, data are presented as geometric mean and $95 \%$ confidence interval. ${ }^{3,15,16}$ In secondary analyses, we also used a RMANOVA to assess treatment $\times$ time effects on weight adjusting (covariate) for study entry BMI, and explored whether changes in urinary BPA concentrations were associated with weight changes. Significance was set at $\alpha<0.05$. Summary statistics are reported as mean (SD) for participant characteristics; geometric mean, change in geometric mean, and 95\% confidence interval for urinary BPA concentrations; and mean \pm SE for creatinine concentrations and body weight.

\section{Results}

Participant characteristics are presented in Table 1, with no difference between groups. All women were non-Hispanic White, never married, college students. No women were lost to follow-up.

Table 2 presents geometric mean and $95 \%$ confidence interval urinary BPA concentrations and NHANES 20092010 reference values in women. ${ }^{19}$ There was a significant treatment $\times$ time effect on creatinine-adjusted urinary BPA concentrations $(p<0.05)$. From study entry to 3 weeks, geometric mean urinary BPA concentrations significantly declined by $0.71 \mathrm{ng} / \mathrm{mL}$ in the intervention group and significantly increased by $0.32 \mathrm{ng} / \mathrm{mL}$ in the control group $(p<0.05)$. There was no significant treatment $\times$ time effect $(p>0.05)$ observed from study entry to 3 weeks on urinary creatinine concentrations in intervention (181 \pm 23 and $159 \pm 28 \mathrm{mg} / \mathrm{dL}$, respectively) and control (168 \pm 25 and $175 \pm 33$, respectively) groups. In sensitivity analyses, removing creatinine concentrations as a fixed effect in our model did not alter group differences in BPA concentrations (data not shown). A significant treatment $\times$ time effect was also observed on group changes in weight $(p<0.05)$. From study entry to 3 weeks, weight significantly declined in the intervention $(-0.28 \pm 0.44 \mathrm{~kg})$, but increased in the control $(1.65 \pm 0.74 \mathrm{~kg})$ group. Changes in creatinine-adjusted BPA concentrations and weight were not significantly related $(p=0.67)$.

\section{Discussion}

In this pilot study, a 3-week behavioral intervention to decrease BPA exposure significantly reduced urinary BPA concentrations relative to a control group in women. Geometric mean urinary BPA declined by $0.71 \mathrm{ng} / \mathrm{mL}$ in the intervention, but increased by $0.32 \mathrm{ng} / \mathrm{mL}$ in controls, underscoring the short-term efficacy of an intervention to decrease urinary BPA in normal-weight, college-aged women. However, given the relatively small sample size of this pilot study, results should be interpreted with some caution as large-scale clinical trials are warranted to confirm these findings.

Few other studies have examined interventions to reduce $\mathrm{BPA}$, and none in women alone, who typically are exposed to higher BPA concentrations. ${ }^{3}$ One nonrandomized study found significant reductions in BPA after a 3-day "fresh food" diet. ${ }^{15}$ Another 5-day randomized dietary replacement study showed an actual increase in BPA, ${ }^{16}$ but food provided to the intervention group was DEHP contaminated and possibly also had BPA contamination. The current study was, by comparison, longer term ( 3 weeks) and tested an intervention that included daily self-monitoring of BPA exposures and provision of BPA-free products strictly in women, with a reduction of $0.71 \mathrm{ng} / \mathrm{mL}$ in geometric mean urinary BPA concentrations. Based on cross-sectional and epidemiological data, this magnitude of reduction would reduce the risk for cardiovascular disease and type 2 diabetes. ${ }^{20,21}$ However, future experimental studies are needed to determine whether there is an actual direct effect of reduced BPA concentrations on risk markers (e.g., lipid panel, insulin, glucose) related to the pathogenesis of cardiovascular disease and type 2 diabetes.

Table 2. Geometric Mean Urinary BPA Concentrations in Control and Intervention Groups, aNd the National Health and Nutrition Examination SURVEY 2009-2010 19 AS REFERENCE VALUE IN WOMEN

\begin{tabular}{|c|c|c|c|c|c|c|}
\hline & \multicolumn{2}{|c|}{ Control } & \multicolumn{2}{|c|}{ Intervention } & \multirow[b]{2}{*}{$\begin{array}{l}\mathrm{p}, \text { Treatment } \times \\
\text { time effect }\end{array}$} & \multirow[b]{2}{*}{$\begin{array}{c}\text { NHANES }{ }^{19} \\
\text { GM }(95 \% \text { CI) }\end{array}$} \\
\hline & $\begin{array}{c}\text { Stlddy entry } \\
\text { GM }(95 \% \text { CI })\end{array}$ & $\begin{array}{c}\text { 3-Weeks } \\
\text { GM (95\% CI) }\end{array}$ & $\begin{array}{c}\text { Study entry } \\
G M(95 \% C I)\end{array}$ & $\begin{array}{c}\text { 3-Weeks } \\
\text { GM (95\% CI) }\end{array}$ & & \\
\hline $\mathrm{BPA}(\mathrm{ng} / \mathrm{mL})$ & $1.06(0.67,1.66)$ & $1.37(0.87,2.15)$ & $1.59(1.05,2.41)$ & $0.88(0.58,1.34)$ & $0.04^{\mathrm{a}}$ & $1.73(1.60,1.87)$ \\
\hline
\end{tabular}

\footnotetext{
${ }^{a}$ Significantly different based on repeated measures analysis of variance using urinary creatinine concentrations and body mass index as fixed effect, and adjusting for study entry BPA concentrations.

BPA, bisphenol A; NHANES, National Health and Nutrition Examination Survey.
} 
In exploratory analyses, the intervention also significantly reduced 3-week weight gain. While intervention participants remained relatively weight stable $(-0.28 \mathrm{~kg})$, the control group gained an average of $1.65 \mathrm{~kg}$ over 3 weeks (which was not attributable to outlier weight changes). However, these data need to be interpreted with caution as in an effort to increase awareness of BPA-containing food packaging, the intervention encouraged self-monitoring of food intake with daily diaries. This may have unintentionally led to decreased food intake and explain the group differences in weight changes, and potentially a reduction in urinary BPA by participants eating less food. A recent review showed that self-monitoring was a strong predictor of both shortand long-term weight control. ${ }^{22}$ Future research is needed with detailed measures of diet and designs that account for the potential influence of self-monitoring on caloric intake. Intervention-related changes in BPA were not significantly related to changes in weight. This could reflect limited statistical power in the current study. Future randomized trials are needed that are specifically designed to untangle the relationship, if any, between BPA exposure and weight changes.

Strengths of this study include a randomized design, comprehensive 3-week intervention intended to decrease BPA concentrations, and use of behavioral strategies (selfmonitoring, goals setting, feedback) to reduce BPA exposure. Limitations of the study are the relatively small sample size and, even though this was a randomized controlled trial, we recruited a convenience sample of women from California Polytechnic State University. Thus, all participants were college students who were normal-weight, nonHispanic women, and our findings may not be generalizable to the population at large. Similarly, whether comparable results would occur in people who are overweight/obese individuals remain unclear. Also, urinary BPA and creatinine concentrations are variable from day-to-day ${ }^{23}$ and may be influenced by recent dietary intake. ${ }^{18}$ To minimize the recent dietary influence on urinary BPA and creatinine concentrations in this pilot study, we took great care in collecting fasting urine samples, but future large-scale clinical studies are needed to confirm our positive results collecting repeat urine samples over multiple days. Finally, we did not assess other endocrine disruptors with known negative health consequences. ${ }^{24,25}$ Thus, future intervention studies with longer-term follow-ups and assessment of a broader range of exposures (e.g., bis (2-ethylhexyl) phthalate, dibutyl phthalate, butyl benzyl phthalate, etc.) are now needed to determine whether interventions can effectively reduce human exposure to other endocrine-disrupting chemicals.

\section{Conclusion}

In conclusion, the results of this pilot study showed that a 3-week intervention to reduce BPA exposure significantly decreased urinary BPA concentrations in normal-weight, college-aged women. Future large-scale clinical randomized trials in women are needed to confirm these findings, and to determine whether reducing exposures to endocrinedisrupting chemicals has a positive effect on health outcomes, including reducing risk markers in the pathogenesis of cardiovascular disease and type 2 diabetes.

\section{Acknowledgments}

The authors thank Beautycounter and Susan Stenovec for discounting the cosmetic, body, and skincare products used in this study. The authors thank all participants in this study.

\section{Author Disclosure Statement}

No competing financial interests exist.

\section{References}

1. Vandenberg LN, Hauser R, Marcus M, Olea N, Welshons WV. Human exposure to bisphenol A (BPA). Reprod Toxicol 2007;24:139-177.

2. Kang JH, Kondo F, Katayama Y. Human exposure to bisphenol A. Toxicology 2006;226:79-89.

3. Calafat AM, Ye X, Wong LY, Reidy JA, Needham LL. Exposure of the U.S. population to bisphenol A and 4tertiary-octylphenol: 2003-2004. Environ Health Perspect 2008;116:39-44.

4. Alonso-Magdalena P, Morimoto S, Ripoll C, Fuentes E, Nadal A. The estrogenic effect of bisphenol A disrupts pancreatic beta-cell function in vivo and induces insulin resistance. Environ Health Perspect 2006;114:106-112.

5. Rubin BS, Murray MK, Damassa DA, King JC, Soto AM. Perinatal exposure to low doses of bisphenol A affects body weight, patterns of estrous cyclicity, and plasma LH levels. Environ Health Perspect 2001;109:675-680.

6. Somm E, Schwitzgebel VM, Toulotte A, et al. Perinatal exposure to bisphenol a alters early adipogenesis in the rat. Environ Health Perspect 2009;117:1549-1555.

7. Teppala S, Madhavan S, Shankar A. Bisphenol A and metabolic syndrome: Results from NHANES. Int $\mathbf{J}$ Endocrinol 2012;2012:598180.

8. Shankar A, Teppala S, Sabanayagam C. Urinary bisphenol a levels and measures of obesity: Results from the national health and nutrition examination survey 2003-2008. ISRN Endocrinol 2012;2012:965243.

9. Shankar A, Teppala S, Sabanayagam C. Bisphenol A and peripheral arterial disease: Results from the NHANES. Environ Health Perspect 2012;120:1297-1300.

10. Silver MK, O'Neill MS, Sowers MR, Park SK. Urinary bisphenol A and type-2 diabetes in U.S. adults: Data from NHANES 2003-2008. PLoS One 2011;6:e26868.

11. Jukic AM, Calafat AM, McConnanghey DR, et al. Urinary concentrations of phthalate metabolites and bisphenol $A$ and associations with follicular-phase length, luteal-phase length, fecundability, and early pregnancy loss. Environ Health Perspect 2016;124:321-328.

12. Casey MF, Neidell M. Disconcordance in statistical models of bisphenol $\mathrm{A}$ and chronic disease outcomes in NHANES 2003-08. PLoS One 2013;8:e79944.

13. Olsen L, Lind L, Lind PM. Associations between circulating levels of bisphenol $\mathrm{A}$ and phthalate metabolites and coronary risk in the elderly. Ecotoxicol Environ Saf 2012; 80:179-183.

14. LaKind JS, Goodman M, Naiman DQ. Use of NHANES data to link chemical exposures to chronic diseases: A cautionary tale. PLoS One 2012;7:e51086.

15. Rudel RA, Gray JM, Engel CL, et al. Food packaging and bisphenol $\mathrm{A}$ and bis(2-ethyhexyl) phthalate exposure: Findings from a dietary intervention. Environ Health Perspect 2011;119:914-920.

16. Sathyanarayana $S$, Alcedo $G$, Saelens $B E$, et al. Unexpected results in a randomized dietary trial to reduce 
phthalate and bisphenol A exposures. J Expo Sci Environ Epidemiol 2013;23:378-384.

17. Hamer M, Bell JA, Sabia S, Batty D, Kivimaki M. Stability of metabolically healthy obesity over 8 years: The English Longitudinal Study of Ageing. Eur J Endocrinol 2015;173: 703-708.

18. Barr DB, Wilder LC, Caudill SP, Gonzalez AJ, Needham LL, Pirkle J. Urinary creatinine concentrations in the U.S. population: Implications for urinary biologic monitoring measurements. Environ Health Perspect 2005;113: $192-200$.

19. Fourth Report on Human Exposure to Environmental Chemicals, Updated Tables. Atlanta, GA: U.S. Department of Health and Human Services, Centers for Disease Control and Prevention. www.cdc.gov/exposurereport/, accessed February 1, 2015.

20. Tai X, Chen Y. Urinary bisphenol A concentrations positively associated with glycated hemoglobin and other indicators of diabetes in Canadian men. Environ Res 2016; 147:172-178.

21. Han C, Hong YC. Bisphenol A, hypertension, and cardiovascular diseases: Epidemiological, laboratory, and clinical trial evidence. Curr Hypertens Rep 2016;18:11.
22. Teixeira PJ, Carraca EV, Marques MM, et al. Successful behavior change in obesity interventions in adults: A systematic review of self-regulation mediators. BMC Med 2015;13:84.

23. Ye X, Wong LY, Bishop AM, Calafat AM. Variability of urinary concentrations of bisphenol $\mathrm{A}$ in spot samples, first morning voids, and 24-hour collections. Environ Health Perspect 2011;119:983-988.

24. Yaghjyan L, Sites S, Ruan Y, Chang SH. Associations of urinary phthalates with body mass index, waist circumference and serum lipids among females: National Health and Nutrition Examination Survey 1999-2004. Int J Obes 2015; 39:994-1000.

25. Singh S, Li SS. Bisphenol A and phthatates exhibit similar toxicogenomics and health effects. Gene 2012;494:85-91.

Address correspondence to:

Todd Hagobian, PhD

Department of Kinesiology

California Polytechnic State University

1 Grand Avenue

San Luis Obispo, CA 93407

E-mail: thagobia@calpoly.edu 